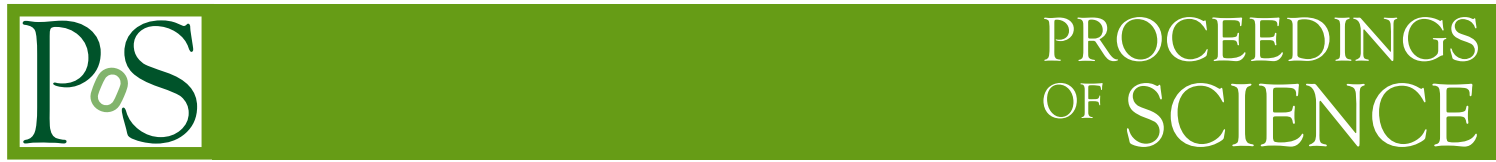

\title{
Equation of State of Gluon Plasma from Gribov Region
}

\section{Daniel Zwanziger*十}

Physics Department, New York University, New York, NY 10003, USA

E-mail: daniel.zwanziger@nyu.edu

We describe the gluon plasma in zeroth order as a gas of free quasi-particles with a temperatureindependent dispersion relation of Gribov type, $E(k)=\sqrt{k^{2}+\frac{M^{4}}{k^{2}}}$, that results from the restriction of the physical state space to the Gribov region. The effective mass $\frac{M^{2}}{k}$ controls infrared divergences of finite-temperature perturbation theory. The equation of state of this gas is calculated and compared with numerical lattice data. At high temperature $T$ there are power corrections to the Stefan-Boltzmann law that are of relative order $1 / T^{3}$ for pressure and $1 / T^{4}$ for energy.

XXIIIrd International Symposium on Lattice Field Theory

25-30 July 2005

Trinity College, Dublin, Ireland

\footnotetext{
* Speaker.

${ }^{\dagger}$ Research supported in part by the National Science Foundation, Grant No. PHY-0099393.
} 


\section{Introduction}

The equation of state (EOS) of the gluon plasma is rather precisely known from numerical studies in lattice gauge theory [1], and there exist excellent phenomenological fits to the EOS of the gluon plasma in the deconfined phase [2]. In contrast with this practical success, it was discovered by Linde [3] as far back as 1980 that finite-temperature perturbative QCD suffers at a fundamental level from infrared divergences, which suggests that finite-temperature perturbation theory neglects an essential feature of QCD. It has been proposed [4] to control these divergences by introducing a magnetic mass $m \sim g^{2} T$. It has also been proposed [5] that the divergences stem from an inadequate application of the principle of gauge equivalence. Indeed in 1978, two years before Linde's discovery of the infrared divergence [3], Gribov showed that infrared modes are strongly suppressed when gauge equivalence is imposed at the non-perturbative level [6].

Two or more different configurations may be gauge equivalent even though both satisfy the linear Coulomb gauge condition, $\sum_{i=1}^{3} \partial_{i} A_{i}=0$. When enumerating physical states, only one of these "Gribov" or gauge copies should be counted, so the space of physical states is reduced to the fundamental modular region (FMR), a region that is free of Gribov copies. Gribov [6] found that the dispersion relation $E(k)=k$ gets modified because of the reduction of the physical state space, and he obtained instead

$$
E(k)=\sqrt{k^{2}+\frac{M^{4}}{k^{2}}}
$$

where $k=|\mathbf{k}|$, and $M$ is a QCD mass scale. The reduction of the physical state space was originally proposed as an essential feature of the confinement mechanism [6, 7]. However statistical mechanics is primarily a matter of counting states, and the reduction of the physical state space required by the gauge principle influences the EOS at all temperatures. Here we shall be concerned with its effect in the deconfined phase.

It is known from numerical studies [1] that at high temperature the EOS of the gluon plasma approaches the Stefan-Boltzmann law, $\varepsilon=3 p=3 c_{S B} T^{4}$, where $\varepsilon$ is the energy per unit volume, $p$ is the pressure, $T$ is the temperature, and $c_{\mathrm{SB}}=\frac{\pi^{2}}{45}\left(N^{2}-1\right)$ in $\mathrm{SU}(\mathrm{N})$ gauge theory. Thus it seems reasonable to describe the gluon plasma at high temperature in first approximation as a gas of noninteracting quasi-particles. We shall describe the quasi-particles by the Gribov dispersion relation (1.1) or a similar one, for $E(k)$ is only approximately known. Fortunately the results obtained hold under rather general conditions on $E(k)$. We call the gas of non-interacting quasi-particles, with modified dispersion relation, the FMR gas.

Many aspects of the Gribov scenario have been verified in recent investigations. Infrared suppression of the gluon propagator in Coulomb gauge has been observed in numerical simulation [8], but less strongly in [9], and in Landau gauge in 3-dimensions [10]. It has also been found in variational calculations in Coulomb gauge [11], and in Schwinger-Dyson calculations in Coulomb [12] and in Landau [13] gauge. A long-range color-Coulomb potential was found in numerical simulations in Coulomb gauge in the deconfined phase [14], and is reported in the talk by Stefan Olejník at this conference.

The questions we wish to address here are: (i) What is the equation of state of the FMR gas? (ii) How does it compare with the EOS that is known from numerical studies? (More details are provided in [15].) 


\section{EOS of the FMR gas}

In the Stefan-Boltzmann limit, the degrees of freedom for each gluon momentum $\mathbf{k}$ are the two states of polarization, each with color multiplicity $\left(N^{2}-1\right)$. These are precisely the degrees of freedom in Coulomb gauge, and we shall use this gauge for our calculation. It is a "physical" gauge without negative metric states, and all constraints are satisfied identically. Although the Coulomb gauge is not manifestly Lorentz covariant, this is not necessarily a disadvantage in the deconfined phase because at finite $T$ the heat bath provides a preferred Lorentz frame, and the manifest symmetries of the Coulomb gauge are the symmetries of the physical problem at hand.

The partition function of the FMR gas is given by the Planck distribution, $Z=\prod_{n}[1-$ $\left.\exp \left(-\beta E_{n}\right)\right]^{-1}$, where $\beta=1 / T$ is the inverse temperature, and $n=(\mathbf{k}, \lambda, a)$, where $\mathbf{k}$ is 3-momentum, $\lambda=1,2$ is polarization, and $a=1, \ldots\left(N^{2}-1\right)$ is color. For the energy density, $\varepsilon=-\frac{1}{V} \frac{\partial \ln Z}{\partial \beta}$, one obtains

$$
\varepsilon=\frac{\left(N^{2}-1\right)}{\pi^{2}} \int_{0}^{\infty} d k \frac{k^{2}}{\exp [\beta E(k)]-1} E(k)
$$

and for the pressure, $p=T \frac{\partial \ln Z}{\partial V}=\frac{T}{V} \ln Z$,

$$
p=\frac{\left(N^{2}-1\right)}{3 \pi^{2}} \int_{0}^{\infty} d k \frac{k^{3}}{\exp [\beta E(k)]-1} \frac{\partial E(k)}{\partial k},
$$

and for the trace anomaly $\theta \equiv \varepsilon-3 p$,

$$
\theta=\frac{\left(N^{2}-1\right)}{\pi^{2}} \int_{0}^{\infty} d k \frac{k^{4}}{\exp [\beta E(k)]-1} \frac{\partial}{\partial k}\left(\frac{-E(k)}{k}\right)
$$

We also have $\varepsilon=T^{2} \frac{\partial}{\partial T}\left(\frac{p}{T}\right)$, from which it follows that the trace anomaly may be written $\theta=$ $\varepsilon-3 p=T^{5} \frac{\partial}{\partial T}\left(\frac{p}{T^{4}}\right)$. Upon integration this yields

$$
p=c_{\mathrm{SB}} T^{4}-T^{4} \int_{T}^{\infty} d T^{\prime} T^{\prime-5} \theta\left(T^{\prime}\right) .
$$

\section{FMR gas at high temperature}

Suppose that the leading deviation of $E(k)$ from $k$ at high $k$ is expressed by a power law,

$$
E(k) / k=1+c / k^{\gamma}+\ldots .
$$

For the Gribov dispersion relation $E(k)=\sqrt{k^{2}+M^{4} / k^{2}}$ one has $\gamma=4$, whereas for a gluon mass, $E(k)=\sqrt{k^{2}+m^{2}}$, one has $\gamma=2$. The gluon condensate has dimension 4, which leads one to expect $\gamma=4$, whereas if there were a condensate of dimension 2, one would expect $\gamma=2$. The asymptotic behavior of the EOS is qualitatively different for $\gamma$ greater or less than 3. We suppose $\gamma>3$, and consequently the deviation from the ultraviolet behavior $E(k)=k$ is soft.

For $\gamma>3$, the asymptotic high- $T$ limit of the trace anomaly is obtained from the substitution $\exp \left[\frac{E(k)}{T}\right]-1 \rightarrow \frac{E(k)}{T}$ in (2.3). This substitution cannot be made in the integrals for $p$ and $\varepsilon$ because they would diverge. It gives a linear asymptotic trace anomaly,

$$
\theta=L T+O(1)
$$


where

$$
\begin{aligned}
L & =\frac{\left(N^{2}-1\right)}{\pi^{2}} \int_{0}^{\infty} d k \frac{k^{4}}{E(k)} \frac{\partial}{\partial k}\left(\frac{-E(k)}{k}\right) \\
& =3\left(N^{2}-1\right) \pi^{-2} \int_{0}^{\infty} d k k^{2} \ln [E(k) / k],
\end{aligned}
$$

is an integral that converges for $\gamma>3$ (as we have supposed), and is positive for $E(k)>k$. These are sufficient conditions for the linear asymptotic form (3.2) with $L>0$. Although $L$ describes the high- $T$ limit of the FMR gas, the last integral involves all momenta $k$. For the special case of the Gribov dispersion relation (1.1), one obtains $L=\left(N^{2}-1\right)(\pi \sqrt{2})^{-1} M^{3}$, which is proportional to $M^{3}$ although only $M^{4}$ appears in the dispersion relation.

The pressure at high $T$ is obtained from (2.4) which yields

$$
p=c_{S B} T^{4}-(1 / 3) L T+O(1)
$$

Thus for the FMR gas, the leading deviation of the pressure from the Stefan-Boltzmann law is linear in $T$. However this linear term - and only a linear term — is annihilated in the formula for the energy density $\varepsilon=T^{2} \frac{\partial}{\partial T}\left(\frac{p}{T}\right)$, which gives

$$
\varepsilon=3 c_{S B} T^{4}+O(1)
$$

An EOS of this type was obtained as a fit to the lattice data in [16].

\section{Comparison with numerical EOS}

The EOS of the FMR gas at high $T$ is not sensitive to the exact form of $E(k)$ because (3.4) and (3.5) hold as long as $\gamma>3$ and $E(k) \geq k$. To compare with the numerical data, we take the Gribov dispersion relation (1.1). The unknown mass scale $M$ is determined by fitting the anomaly $\theta=\varepsilon-3 p$ at high $T$, because the corrections to this quantity are expected to be small. [Indeed, taking thermal perturbation theory as a guide [17], we note that the leading correction to $p$ is of order $\Delta p \sim g^{2}(T) T^{4} \sim \frac{T^{4}}{\ln T}$. The anomaly is given by $\theta=T^{5} \frac{\partial}{\partial T}\left(\frac{p}{T^{4}}\right)$, so the corresponding correction to the anomaly, $\Delta \theta=T^{5} \frac{\partial}{\partial T}\left(\frac{\Delta p}{T^{4}}\right)$, is of order $T^{5} \frac{\partial}{\partial T}\left(\frac{1}{\ln T}\right)=-\frac{T^{4}}{\ln ^{2} T} \sim g^{4}(T) T^{4}$.]

The numerical data of [1] for $\frac{\theta}{T^{4}}$ are represented by the black interpolating curves in Fig. 1 (left). (More recent studies include dynamical fermions or a chemical potential that cannot be described by the FMR gas.) The data for $N_{\tau}=6$ and 8 agree, and were interpreted as continuum values [1]. The red dots are obtained from the analytic formula (2.3), with mass scale set at $M=2.6 T_{c}$ by fitting at high $T$, where $T_{c}$ is the transition temperature. The relatively large difference in the transition region between the FMR gas and the numerical data for $\theta=\varepsilon-3 p$ occurs because the FMR gas does not exhibit a sharp phase transition, whereas for pure $\mathrm{SU}(3)$ gauge theory there is a first order phase change, so $\varepsilon$ is discontinuous while $p$ is continuous. We do not attempt to estimate the error of $M$ because perturbative-type corrections to the FMR gas have been neglected. From the value $T_{c}=0.625 \sqrt{\sigma}$ of [1], where $\sigma$ is the string tension, one gets $M=1.6 \sqrt{\sigma}$, or $M=705 \mathrm{MeV}$, where the string tension for the quarkless theory is defined to be $\sqrt{\sigma}=440 \mathrm{MeV}$.

The numerical data of [1] for $\frac{\varepsilon}{T^{4}}, \frac{3 s}{4 T^{4}}$ and $\frac{3 p}{T^{4}}$ are are displayed as black interpolating curves in Fig. 1 (right), where $\frac{s}{T^{3}}=\frac{\varepsilon+p}{T^{4}}$. The horizontal line represents the Stefan-Boltzmann EOS. For the 

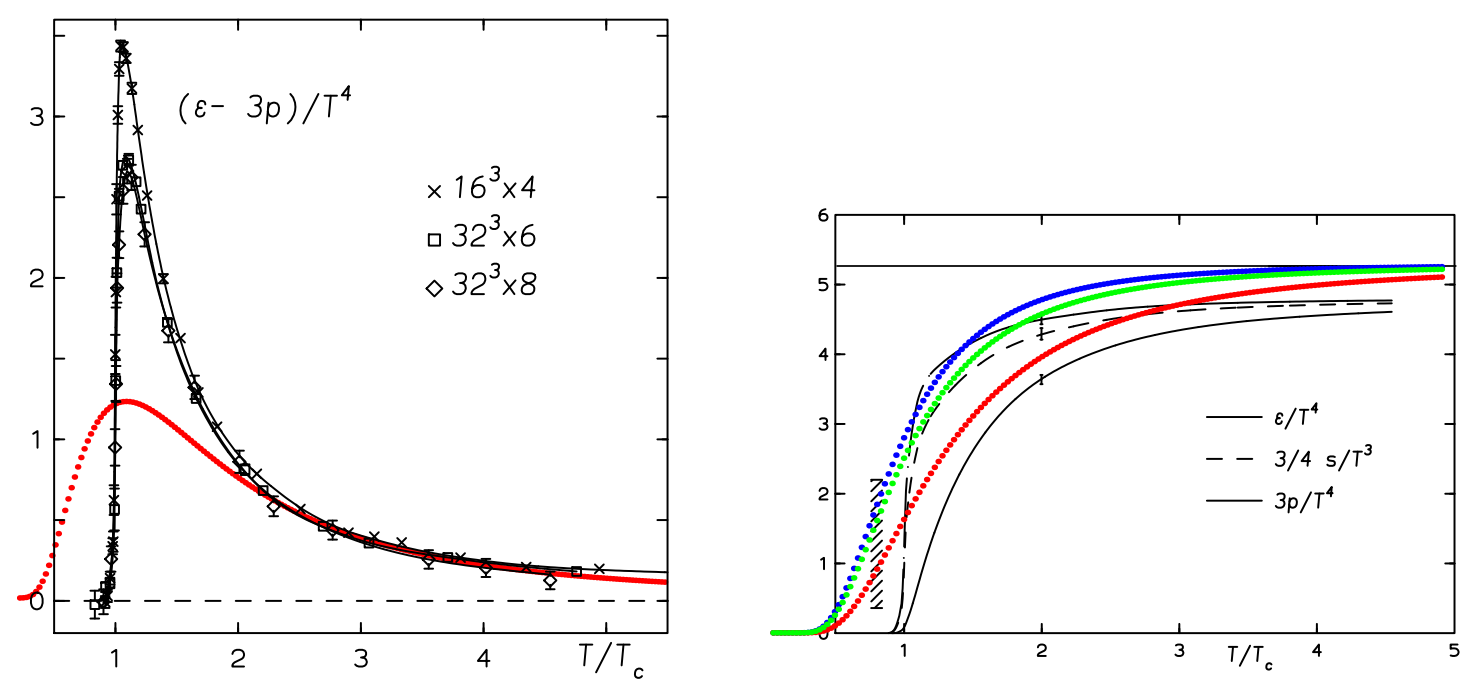

Figure 1: Numerical and analytic plots of $(\varepsilon-3 p) / T^{4}$ (left), and of $\varepsilon / T^{4}, 3 / 4 s / T^{3}, 3 p / T^{4}$ (right).

FMR gas, $\frac{3 p}{T^{4}}$ and $\frac{\varepsilon}{T^{4}}$ approach the Stefan-Boltzmann limit like $\frac{1}{T^{3}}$ and $\frac{1}{T^{4}}$ respectively, being quite close to it at the highest temperature displayed, $T=5 T_{c}$, whereas the gluon plasma approaches the Stefan-Boltzmann limit more slowly. The difference between the FMR gas and the gluon plasma in the range $2 T_{c}$ to $5 T_{c}$ appears attributable to perturbative-type corrections of moderate size. In standard thermal perturbation theory [17] these are of leading order $g^{2}(T) \sim \frac{1}{\ln T}$, but diverge at order $g^{6}$, whereas corrections to the FMR gas are expected to be calculable.

\section{Discussion}

Above the transition region, the EOS of the FMR gas gives a good description of the most prominent feature of the gluon plasma which is the rapid drop of the pressure compared to the energy from the Stefan-Boltzmann value, as $T$ decreases from infinity. The linear asymptotic trace anomaly (3.2) provides a ready explanation for this, that holds for any quasi-particle model with $\gamma>3$ and $\frac{\partial}{\partial k}\left(\frac{E(k)}{k}\right)<0$, although other fits are certainly not excluded. The FMR gas is not exact even at high temperature because of perturbative-type corrections. We expect however that they are calculable and of moderate size above the transition region.

The transition region is not so well described by the FMR gas. There is no sharp phase transition because $p(T)$ is an analytic function. Moreover the dependence on $\mathrm{N}$ [of $\mathrm{SU}(\mathrm{N})$ ] is only through the coefficient $\left(N^{2}-1\right)$, whereas even the order of the phase change depends on $N$, being second order for SU(2) and first order for SU(3). An analysis of the phase transition based on center symmetry is given in [18]. However the dispersion relation $E(k)=\sqrt{k^{2}+\frac{M^{4}}{k^{2}}}$ has a minimum energy $E_{\min }=\sqrt{2} M$, so the thermodynamic functions $\varepsilon, p$ and $s$ of the FMR gas are exponentially small for $T<\sqrt{2} M=997 \mathrm{MeV}$ (for $M=705 \mathrm{MeV}$ ). The mass of the lightest glueball is of order $1 \mathrm{GeV}$, so the thermodynamic functions of the FMR gas are exponentially small where they are 
supposed to be. Altogether the FMR gas, with a single parameter $M$ which is the mass scale, is competitive with cut-off phenomenological models [19].

The improved phenomenological models of [2] are more precise than the FMR gas especially in the transition region, but they also require several parameters, whereas we have fit only the QCD mass scale $M$. However the FMR gas is not intended to be a precise phenomenological model, but rather to provide a useful starting point, well founded in the principles of gauge theory, that allows calculable, moderate size corrections at high $T$. Athough it is defined by Gribov's dispersion relation of 1978, the FMR gas has two important properties that were later independently reinvented: (i) Its EOS closely resembles simple phenomenological models of the gluon plasma [19]. (ii) The

effective mass $\frac{M^{2}}{k}$ controls infrared divergences in higher order corrections so it is not necessary to introduce the magnetic mass $m \sim g^{2} T$ [4] for this purpose.

\section{References}

[1] G. Boyd, J. Engels, F. Karsch, E. Laermann, C. Legeland, M. Lütgemeier and B. Petersson, Phys. Rev. Lett. 75, 4169 (1995) and Nucl. Phys. B 469419 (1996).

[2] J. Engels, J. Fingberg, K. Redlich, H. Satz, M. Weber, Z. Phys. C 42341 (1989); A. Peshier, B. Kämpfer, O. P. Pavlenko, and G. Soff, Phys. Rev. D 54, 2399 (1996); Peter Levai and Ulrich Heinz, Phys. Rev. C 571879 (1998); R. A. Schneider and W. Wiese, Phys. Rev. C 64055201.

[3] A. D. Linde, Phys. Lett. B 96, 289, (1980)

[4] D. Gross, R. Pisarski, L. Yaffe, Rev. Mod Phys. 53, 43 (1981).

[5] I. Zahed and D. Zwanziger, Phys. Rev. D 61, 037501 (2000).

[6] V. Gribov, Nucl. Phys. B 139, 1 (1978); D. Zwanziger, Nucl. Phys. B 485185 (1997).

[7] Richard P. Feynman, Nucl. Phys. B188 479 (1981).

[8] A. Cucchieri and D. Zwanziger, Phys. Rev. D 65, 014001 (2001).

[9] K. Langfeld and L. Moyaerts, arXiv: hep-lat/0406024.

[10] A. Cucchieri, T. Mendes and A. Taurines, Phys. Rev. D 67, 091502 (2003).

[11] A. Szcepaniak, arXiv: hep-ph/0306030; C. Feuchter, H. Reinhardt, Phys. Rev. D70 (2004) 105021; H. Reinhardt, C. Feuchter, arXiv: hep-th/0408237.

[12] D. Zwanziger, arXiv: hep-ph/0312254.

[13] L. von Smekal, A. Hauck and R. Alkofer, Ann. Phys. 267, 1 (1998); L. von Smekal, A. Hauck and R. Alkofer, Phys. Rev. Lett. 79, 3591 (1997); C. Lerche and L. von Smekal, arXiv: hep-ph/0202194; D. Zwanziger, Phys. Rev. D 65, 094039 (2002).

[14] J. Greensite, Š. Olejník, and D. Zwanziger, Phys. Rev. D 69, 074506 (2004), arXiv: hep-lat/0401003.

[15] D. Zwanziger, Phys. Rev. Lett. 94,182301(2005), arXiv: hep-ph/0407103.

[16] C. G. Källman, Phys. Lett. 134B, 363 (1984).

[17] J. I. Kapusta, Nucl. Phys. B148, 461 (1979).

[18] R. Pisarski, Phys. Rev. D62 111501 (2000).

[19] T. DeGrand and C. DeTar, Phys. Rev. D 35742 (1987); F. Karsch, Z. Phys. C 38147 (1988);

D. Rischke, M. Gorenstein, A. Schäfer, H. Stöcker, W. Greiner, Phys. Letts. B 27819 (1992). 